\title{
Procalcitonin in bloodstream infections: beyond its role as a marker of clinical algorithm to reduce antimicrobial overuse
}

\author{
Yong Kyun Kim
}

Division of Infectious Diseases, Department of Internal Medicine, Inje University Haeundae Paik Hospital, Busan, Korea

Received: December 8, 2017 Accepted: December 14, 2017

\section{Correspondence to}

Yong Kyun Kim, M.D.

Division of Infectious Diseases, Department of Internal Medicine, Inje University Haeundae Paik Hospital, 875 Haeun-daero, Haeundae-gu, Busan 48108, Korea

Tel: +82-51-797-0316

Fax: +82-51-797-3229

E-mail: amoureuxyk@naver.com

\section{See Article on Page 176-184}

To improve clinical outcomes of sepsis through more timely diagnosis and treatment, several biological markers for sepsis-specific clinical manifestations have been extensively evaluated [1]. However, only a few potential biomarkers for the diagnosis of sepsis have been appropriately studied, and the majority are not routinely used in clinical practice because of limited specificity or sensitivity [2].

One potential biomarker for the diagnosis of sepsis is procalcitonin (PCT). During sepsis, PCT is synthetized massively by various types of cells. In 1993, it was described as accurately differentiating between systemic bacterial infections and noninfectious inflammatory states [3]. Since then, growing evidence has emerged to support PCT as a tool for optimizing antibiotic therapy in patients with sepsis. For example, a recent meta-analysis of eight randomized trials concluded that a PCT-guided discontinuation of antibiotics improved short-term mortality and duration of antibiotic therapy compared to standard care [4]. In this context, recent guidelines recommend the use of PCT levels to shorten the duration of antibiotic therapy for the management of hospital-acquired/ventilator-associated pneumonia and sepsis/septic shock $[5,6]$. However, there is insufficient evidence to support using PCT levels to guide antibiotic initiation and to verify the beneficial effect on short-term mortality and antibiotic consumption [7-9]. Thus, monitoring patients' level of PCT has not generally been considered a way of assisting clinicians in deciding when to initiate antibiotic therapy [5].

Meanwhile, the diagnostic accuracy of PCT in patients with bloodstream infection (BSI) has also been evaluated to diagnose BSI without taking blood cultures. A recent meta-analysis revealed that PCT was useful for ruling out the presence of bacteremia, with a sensitivity of $76 \%$ and specificity of $69 \%$ when a cutoff level of $0.5 \mathrm{ng} / \mathrm{mL}$ was used for diagnosing BSI [10]. However, because a substantial portion of patients with bacteremia can have PCT $<0.5 \mathrm{ng} / \mathrm{mL}$ (37.9\%), the best PCT cutoff value for ruling out bacteremia remains unknown [11]. In addition, potential confounders, such as infection site, renal function, and pathogen categories, can also affect PCT levels in patients with BSI [11-13]. Given the complex relationship between PCT and BSI, further research is required regarding the safety and efficacy of using PCT levels as a single diagnostic 
tool for identifying BSI.

In this issue, Cha et al. [14] investigated the usefulness of PCT as a diagnostic marker in patients with suspected nosocomial BSI and assessed the relationship between PCT and renal function. To compare clinical characteristics and outcomes of patients with nosocomial BSI and a non-BSI group $(n=680)$, the researchers enrolled 105 patients who developed BSI more than $48 \mathrm{~h}$ after admission. The optimal PCT cutoff value for predicting BSI accurately was also assessed. The median serum PCT level was significantly higher in the BSI group than in the non-BSI group ( 0.65 and $0.22 \mathrm{ng} / \mathrm{mL}$, respectively; $p=0.001$ ) when an optimal cutoff value of $0.27 \mathrm{ng} / \mathrm{mL}$ was used. The area under the curve (AUC) of PCT (0.692) was also significantly larger than the AUC of C-reactive protein (CRP; 0.526) and white blood cell (WBC) count (0.518). This indicates that PCT was a more useful diagnostic marker for predicting nosocomial BSI than CRP or WBC count. More important, Cha et al. [14] suggested that using PCT levels as a single diagnostic tool for predicting nosocomial BSI and avoiding taking blood cultures is questionable because PCT could not rule out $26 \%$ of nosocomial BSI patie nts at the optimal cutoff value of $0.27 \mathrm{ng} / \mathrm{mL}$. In addition, it was unable to predict $6 \%$ of nosocomial BSI patients at a lower PCT cutoff value of $0.1 \mathrm{ng} / \mathrm{mL}$. They also noted that the optimal cutoff value of PCT for nosocomial BSI increased along with renal dysfunction $(0.12 \mathrm{ng} / \mathrm{mL}$ with estimated glomerular filtration rate $[\mathrm{eGFR}] \geq 60 \mathrm{~mL} / \mathrm{min} / 1.73$ $\mathrm{m}^{2} ; 0.84 \mathrm{ng} / \mathrm{mL}$ with $30 \leq \mathrm{eGFR}<60 \mathrm{~mL} / \mathrm{min} / 1.73 \mathrm{~m}^{2} ; 1.71$ $\mathrm{ng} / \mathrm{mL}$ with eGFR $<30 \mathrm{~mL} / \mathrm{min} / 1.73 \mathrm{~m}^{2}$ ).

This article has important clinical implications for future patient management. First, the results of the study [14] contribute to growing evidence on this topic. There have been only two meta-analyses of the accuracy of PCT for diagnosing bacteremia, and these studies had conflicting results $[10,15]$. Furthermore, little is known regarding the utility of PCT as a diagnostic assay for bacteremia, particularly in the setting of nosocomial BSI. The results of this article [14] suggest that the efficacy of using PCT as a single diagnostic tool for predicting BSI remains to be proven. Moreover, PCT should be interpreted in the context of clinical situations until well-designed studies with larger patient cohorts strengthen and validate the diagnostic use of PCT in a BSI setting. Second, this study [14] expands results to patients with renal dysfunction and finds that the cutoff value of PCT for identifying sepsis was higher in patients with impaired renal function, which is consistent with previous studies $[16,17]$. Although there is substantial variability in determining the best PCT cutoff value in patients with renal dysfunction, PCT levels around the standard cutoff value should be interpreted with caution. An investigation of this type may be worthwhile given how PCT levels might be used on clinical suspicion of BSI to meet the needs of patients with impaired renal function who are vulnerable to BSI.

No sepsis-specific biomarkers other than PCT have been evaluated in detail using well-designed, prospective, randomized trials in various settings to develop a clinical algorithm for improving antibiotic therapy. The use of PCT levels as a diagnostic biomarker should be expanded to disease-based antimicrobial stewardship, including BSI. Despite the limitations of the clinical relevance of PCT as a diagnostic marker of BSI, future research on the functional assay sensitivity and the best cutoff value for predicting BSI may improve patient outcomes. In an era of the global spread of resistance to antibiotics, this study could be a meaningful step in guiding and providing innovative ideas for improved clinical algorithms and antimicrobial stewardship protocols.

\section{Conflict of interest}

No potential conflict of interest relevant to this article was reported.

\section{REFERENCES}

1. Pierrakos C, Vincent JL. Sepsis biomarkers: a review. Crit Care 2010;14:R15.

2. Reinhart K, Bauer M, Riedemann NC, Hartog CS. New approaches to sepsis: molecular diagnostics and biomarkers. Clin Microbiol Rev 2012;25:609-634.

3. Assicot M, Gendrel D, Carsin H, Raymond J, Guilbaud J, Bohuon C. High serum procalcitonin concentrations in patients with sepsis and infection. Lancet 1993;341:515-518.

4. Huang HB, Peng JM, Weng L, Wang CY, Jiang W, Du B. Procalcitonin-guided antibiotic therapy in intensive care unit patients: a systematic review and meta-analysis. Ann Intensive Care 2017;7:114.

5. Kalil AC, Metersky ML, Klompas M, et al. Management 
of adults with hospital-acquired and ventilator-associated pneumonia: 2016 Clinical Practice Guidelines by the Infectious Diseases Society of America and the American Thoracic Society. Clin Infect Dis 2016;63:e61-e111.

6. Rhodes A, Evans LE, Alhazzani W, et al. Surviving sepsis campaign: international guidelines for management of sepsis and septic shock: 2016. Intensive Care Med 2017;43:304-377.

7. Jensen JU, Hein L, Lundgren B, et al. Procalcitonin-guided interventions against infections to increase early appropriate antibiotics and improve survival in the intensive care unit: a randomized trial. Crit Care Med 2011;39:2048-2058.

8. Layios N, Lambermont B, Canivet JL, et al. Procalcitonin usefulness for the initiation of antibiotic treatment in intensive care unit patients. Crit Care Med 2012;40:23042309.

9. Bouadma L, Luyt CE, Tubach F, et al. Use of procalcitonin to reduce patients' exposure to antibiotics in intensive care units (PRORATA trial): a multicentre randomised controlled trial. Lancet 2010;375:463-474.

10. Hoeboer SH, van der Geest PJ, Nieboer D, Groeneveld AB. The diagnostic accuracy of procalcitonin for bacteraemia: a systematic review and meta-analysis. Clin Microbiol Infect 2015;21:474-481.

11. Yan ST, Sun LC, Jia HB, Gao W, Yang JP, Zhang GQ. Procalcitonin levels in bloodstream infections caused by dif- ferent sources and species of bacteria. Am J Emerg Med 2017;35:579-583.

12. Nakamura Y, Murai A, Mizunuma M, et al. Potential use of procalcitonin as biomarker for bacterial sepsis in patients with or without acute kidney injury. J Infect Chemother 2015;21:257-263.

13. Oussalah A, Ferrand J, Filhine-Tresarrieu P, et al. Diagnostic accuracy of procalcitonin for predicting blood culture results in patients with suspected bloodstream infection: an observational study of 35,343 consecutive patients (a STROBE-compliant article). Medicine (Baltimore) 2015;94:e1774.

14. Cha JK, Kwon KH, Byun SJ, et al. Clinical value of procalcitonin for suspected nosocomial bloodstream infection. Korean J Intern Med 2018;33:176-184.

15. Jones AE, Fiechtl JF, Brown MD, Ballew JJ, Kline JA. Procalcitonin test in the diagnosis of bacteremia: a meta-analysis. Ann Emerg Med 2007;50:34-41.

16. Contou D, d'Ythurbide G, Messika J, et al. Description and predictive factors of infection in patients with chronic kidney disease admitted to the critical care unit. J Infect 2014;68:105-115.

17. Lee WS, Kang DW, Back JH, Kim HL, Chung JH, Shin BC. Cutoff value of serum procalcitonin as a diagnostic biomarker of infection in end-stage renal disease patients. Korean J Intern Med 2015;30:198-204. 